\title{
Vascular endothelial growth factor inhibitors: investigational therapies for the treatment of psoriasis
}

This article was published in the following Dove Press journal:

Clinical, Cosmetic and Investigational Dermatology

25 September 2013

Number of times this article has been viewed

\author{
Anja K Weidemann' \\ Ania A Crawshaw ${ }^{2}$ \\ Emily Byrne 3 \\ Helen S Young' \\ 'The Dermatology Centre, Salford \\ Royal NHS Foundation Trust, The \\ University of Manchester, Manchester, \\ UK; ${ }^{2}$ Royal Sussex County Hospital, \\ Brighton, UK; ${ }^{3}$ University Hospital \\ of South Manchester, Manchester, UK
}

Correspondence: Helen S Young The Dermatology Centre, Salford Royal NHS Foundation Trust, University of Manchester, Manchester Academic Health Science Centre, Stott Lane, Salford, Manchester, M6 8HD, UK Email helen.s.young@manchester.ac.uk

\begin{abstract}
Psoriasis is a common inflammatory autoimmune condition in which environmental factors and genetic predisposition contribute to the development of disease in susceptible individuals. Angiogenesis is known to be a key pathogenic feature of psoriasis. Local and systemic elevation of vascular endothelial growth factor (VEGF)-A has been demonstrated in the skin and plasma of patients with psoriasis and is known to correlate with improvement following some traditional psoriasis treatments. A number of VEGF inhibitors are licensed for the treatment of malignancies and eye disease and isolated case reports suggest that some individuals with psoriasis may improve when exposed to these agents. The small number of cases and lack of unified reporting measures makes it difficult to draw generalizations and underline the heterogeneity of psoriasis as a disease entity. Though not yet licensed for the treatment of psoriasis in humans, experimental data supports the potential of VEGF inhibitors to influence relevant aspects of human cell biology (such as endothelial cell differentiation) and to improve animal models of skin disease. Given the multi-factorial nature of psoriasis it is unlikely that VEGF inhibitors will be effective in all patients, however they have the potential to be a valuable addition to the therapeutic arsenal in selected cases. Current VEGF inhibitors in clinical use are associated with a number of potentially serious side effects including hypertension, left ventricular dysfunction, and gastrointestinal perforation. Such risks require careful consideration in psoriasis populations particularly in light of growing concerns linking psoriasis to increased cardiovascular risk.
\end{abstract}

Keywords: psoriasis, vascular endothelial growth factor, VEGF, VEGF inhibitor

\section{Introduction}

Psoriasis is a common chronic inflammatory skin disease affecting $1 \%-3 \%$ of the Caucasian population. ${ }^{1}$ Patients with psoriasis have a significant degree of associated psychological morbidity ${ }^{2}$ and extra cutaneous manifestations, such as concomitant psoriatic arthritis (in up to $40 \%$ of patients) ${ }^{1}$ and higher incidence of metabolic syndrome (particularly in those with severe disease). ${ }^{3}$

Psoriasis vulgaris is the commonest disease subtype and is characterized clinically by the presence of thickened erythematous scaly plaques. The histological features of psoriasis are: (1) a dermal and epidermal inflammatory infiltrate; (2) epidermal hyperplasia; (3) abnormal keratinocyte differentiation; and (4) increased dermal vascularity with tortuous capillary loops. ${ }^{4,5}$ Classically, the increased vascularity can be demonstrated clinically by "Auspitz sign" where scraping scale from psoriatic plaques leads to pin-point bleeding.

Originally thought to be a primary disorder of keratinocytes, psoriasis is now recognized as a complex autoimmune disease in which environmental factors and 
genetic predisposition contribute to the manifestation of the disease in susceptible individuals. ${ }^{6}$

T-cells (particularly T-helper-1 and T-helper-17) are hypothesized to be key players in the initiation and maintenance of psoriasis; however, many other cell types are also important. Endothelial cells, dendritic cells, monocytic cells, neutrophils, and keratinocytes also contribute to the complicated cocktail of chemokines and cytokines found in affected individuals. ${ }^{7}$ Elucidation of these pathogenic pathways has resulted in an expansion of the therapeutic arsenal though to date psoriasis remains incurable.

Research into new therapies continues and seeks to assess different parts of the psoriasis pathway including inhibition of angiogenesis. Angiogenesis is a key feature in psoriatic skin which is associated with local and systemic elevation of angiogenic cytokines including vascular endothelial growth factor (VEGF) which fluctuates in line with disease activity. ${ }^{8,9}$ Existing psoriasis treatments such as cyclosporine, ${ }^{10}$ acitretin, ${ }^{9}$ fumeric acid esters, ${ }^{11,12}$ infliximab, ${ }^{13,14}$ and psoralen and ultraviolet A (PUVA) ${ }^{15,16}$ have been shown to inhibit angiogenesis and reduce levels of VEGF as part of their therapeutic effect.

As yet, VEGF inhibitors are not licensed for the management of psoriasis; however, VEGF inhibitors are in widespread use for other conditions including malignant disease ${ }^{17,18}$ and age-related macular degeneration. ${ }^{19,20}$ There are isolated case reports of significant improvement in psoriasis during use of VEGF inhibitors for other conditions ${ }^{21-24}$ and supportive data from animal models. ${ }^{25}$

This article will examine the therapeutic potential of VEGF inhibitors in psoriasis with reference to emerging therapies and existing agents used in other conditions.

\section{Vascular endothelial growth factors (VEGFs)}

Angiogenesis is the formation of new blood vessels from a pre-existing vascular bed and usually occurs during embryogenesis, wound healing, and the endometrial cycle. ${ }^{26}$ Pathological angiogenesis is a hallmark of tumor formation, ${ }^{27}$ but also occurs in degenerative eye disease ${ }^{28}$ and inflammatory conditions, such as joint disease ${ }^{29}$ and psoriasis. ${ }^{8}$

VEGFs play a key role in normal and pathological angiogenesis. ${ }^{30}$ Six family members or subtypes exist: VEGFA, VEGF-B, VEGF-C, VEGF-D, VEGF-E, and placental growth factor (PlGF). ${ }^{31}$ Of these, VEGF-A (initially known as vascular permeability factor) was the first to be discovered and exists as four isoforms (VEGF-A121, VEGF-A165, VEGF-A189, VEGF-A206) ${ }^{32}$ generated by variable splicing of the $V E G F-A$ gene. VEGF-A is found intracellularly and secreted systemically ${ }^{30}$ promoting monocyte activation and chemotaxis, ${ }^{33}$ controlling endothelial cell differentiation and increasing vascular permeability. ${ }^{34}$ VEGF-165 is the most common isoform and the most important for angiogenesis. ${ }^{35}$

VEGFs interact with cell membrane receptors (VEGFRs) to activate intracellular tyrosine kinases. ${ }^{34}$ VEGFRs exist as three subtypes (VEGFR-1, VEGFR-2, and VEGFR-3) and consist of seven extracellular immunoglobulin-like domains and an intracellular tyrosine kinase domain. VEGF-A has a high affinity for VEGFR-1 and VEGFR-2 through which it mediates its biological effects. ${ }^{36}$

In humans, heterozygous and homozygous defects in VEGF-A alleles are fatal. ${ }^{37}$ The $V E G F-A$ gene is highly polymorphic $^{38,39}$ with some polymorphisms (eg, rs2010963 and rs833061) being associated with early onset psoriasis. The VEGF-A gene is in close proximity to PSORSI (a gene strongly associated with psoriasis hereditability) on chromosome 6p21, however, no linkage disequilibrium between the two has been observed suggesting that they are inherited independently. ${ }^{40}$

\section{VEGF-A in psoriasis}

In the skin, VEGF-A is predominantly secreted by keratinocytes. Patients with psoriasis have higher levels of VEGF-A secretion in both affected and non-affected skin with affected skin showing significantly higher levels that fluctuate in line with disease activity. ${ }^{41}$ Plasma levels of VEGF-A are also elevated in patients with psoriasis and fluctuate with disease activity. ${ }^{9,42}$ High plasma levels of VEGF-A are associated with early onset psoriasis (onset before the age of 40 years) and psoriatic arthritis. ${ }^{43}$

In 2003, Xia et $\mathrm{a}^{25}$ noted the development of inflammatory skin lesions in otherwise healthy transgenic VEGF mice. The skin changes were clinically and histologically similar to human psoriasis - including demonstration of the Koebner phenomenon - and were associated with high levels of epidermal, dermal and circulating VEGF. Introduction of a VEGF antagonist led to resolution of the psoriasiform eruption. ${ }^{25}$

In humans, the use of some traditional psoriasis therapies has been associated with reduction of VEGF-A expression. Andrys et al found that use of topical coal tar in combination with ultraviolet B (UVB; Goeckerman therapy) in patients with psoriasis led to significant clinical improvement and reduced plasma levels of VEGF-A.42

These findings are in keeping with in vitro studies, which demonstrate that photochemotherapy with PUVA 
suppresses VEGF expression, inhibits angiogenesis, and induces apoptosis of human endothelial cells ${ }^{15}$ and in vivo studies that showed reduced plasma levels of VEGF-A following PUVA therapy. ${ }^{16}$

However, the relationship between VEGF levels, phototherapy, and therapeutic effect in psoriasis is by no means clear as treatment with narrow-band (NB)-UVB and retinoid (re)-PUVA has been shown to lead to higher levels of VEGFA than at baseline despite clinical improvement. ${ }^{16}$

These seemingly contradictory findings may be explained by increased epidermal proliferation following UVB exposure and individual response to systemic retinoids.

Skin thickening via epidermal hyperplasia is a wellrecognized consequence of UV exposure ${ }^{44}$ and irradiation of normal skin with UVB results in an upregulation of VEGF-A. ${ }^{45}$ Bielenburg et al demonstrated that exposure of $\mathrm{C} 3 \mathrm{H} / \mathrm{HeN}$ mice to a one-off dose of UVB resulted in epidermal hyperplasia and new vessel formation. They found that the proliferating keratinocytes were producing angiogenic cytokines resulting in increased cutaneous angiogenesis. ${ }^{46}$ It is likely that a similar process occurs in irradiated skin of patients undergoing UVB therapy, but that in many patients the balance is still in favor of a beneficial therapeutic effect via other mechanisms.

In the case of re-PUVA, all-trans retinoic acid is reported to have a genotype-dependent inhibitory effect on keratinocyte production of VEGF-A, while also having a genotype-independent stimulatory effect on peripheral blood mononuclear cells which could be used to predict clinical response to acitretin. ${ }^{9}$ Akman et al hypothesized that the seemingly paradoxical increase in VEGF-A levels following re-PUVA therapy could be a rebound phenomenon secondary to exposure to a systemic retinoid where peripheral blood mononuclear cells had been stimulated to produce higher levels of VEGF-A. ${ }^{16}$

Though their exact mechanism of action remains unknown, fumeric acid esters have been shown to inhibit angiogenesis, ${ }^{11}$ impair endothelial cell function, and suppress expression of VEGFR-2. ${ }^{12}$ It has been postulated that in addition to their direct anti-angiogenic properties, their anti-psoriatic effects relate to inhibition of response to circulating VEGF-A. ${ }^{47}$

Cyclosporin A has also been shown to inhibit in vitro and in vivo angiogenesis via inhibition of cyclooxygenase (Cox)-2. ${ }^{10}$ The gene for Cox-2 is induced by VEGF-A, but also differentially regulated by tumor necrosis factor alpha (TNF- $\alpha){ }^{48}$ TNF- $\alpha$ upregulates VEGFR-2 expression and has been shown to stimulate angiogenesis, enhance
VEGF-mediated endothelial cell migration, and enhance wound healing. ${ }^{48}$

TNF- $\alpha$ levels are elevated in psoriasis and play an important part in T-cell proliferation and disease pathogenesis. ${ }^{49}$ Treatment with the anti-TNF- $\alpha$ agent infliximab improves skin and joint disease ${ }^{50}$ and reduces VEGF expression in dermal and synovial tissue with corresponding reductions in tissue vascularity. ${ }^{13,14}$

\section{Current VEGF inhibitors}

As discussed above, many existing psoriasis treatments influence the production or function of VEGF-A. Though as yet there is no agent specifically targeting the VEGF pathway in psoriasis, VEGF inhibitors have been used for the treatment of other conditions, particularly in cancer therapy ${ }^{51}$ and eye disease. ${ }^{52}$

Existing VEGF inhibitors target the VEGF pathway in various ways including: (1) direct inhibition of VEGF protein (anti-VEGF monoclonal antibodies; bevacizumab and ranibizumab); (2) prevention of VEGF receptor binding (VEGF receptor antagonists; alfibercept/VEGF-Trap and pegaptanib); and (3) inhibition of VEGF receptor function through inhibition of tyrosine kinase (tyrosine kinase inhibitors [TKIs]; sunitinib, sorafenib, vandetanib, and pazopanib).

Many new anti-angiogenic therapies are under investigation. These include: ramucirumab, cediranib, nintedanib (BIBF 1120), pazopanib, brivanib, ABT-869, axitinib, ABT-751, and NPI-2358. ${ }^{53}$

It is beyond the scope of this article to review all existing and developing anti-angiogenic drugs in detail; however, examples of different classes of agent are given and particular mention is made of existing agents which have been reported to effect psoriasis. Potential therapies for psoriasis are discussed below under "Emerging therapies in psoriasis".

\section{Anti-VEGF monoclonal antibodies Bevacizumab}

Bevacizumab (Avastin ${ }^{\mathrm{TM}}$; Genentech/Roche, South San Francisco, CA, USA) is a recombinant, humanized VEGFA neutralizing, monoclonal antibody derived from mouse anti-VEGF antibodies. ${ }^{54}$ It was the first anti-angiogenic agent licensed for use in oncology and was licensed by the USFood and Drug Administration (FDA) in 2004 as first-line treatment of metastatic colon cancer.

Bevacizumab is also licensed for the second-line treatment of metastatic colon or rectal cancer, ${ }^{55}$ metastatic non-squamous non-small cell lung carcinoma, ${ }^{56}$ recurrent 
glioblastoma multiforme, ${ }^{55}$ and metastatic renal cell carcinoma. ${ }^{57}$

Though bevacizumab was initially thought to show promise in the treatment of metastatic breast cancer, the FDA withdrew its license after large, randomized Phase III trials failed to show any survival benefit. ${ }^{51}$ Bevacizumab is being investigated in a number of other tumor groups including ovarian cancer where Phase III trials are underway. ${ }^{58}$

Off-license indications for bevacizumab include various ocular conditions (eg, age-related macular degeneration, diabetic retinopathy, radiation retinopathy, neovascular glaucoma, corneal neovascularization, and ocular oncology) with delivery by intravitreal, subconjunctival, intracameral, and intracorneal routes. ${ }^{59}$

\section{Ranibizumab}

Ranibizumab (Lucentis ${ }^{\mathrm{TM}}$; Genentech) is a monoclonal antibody fragment derived from bevacizumab specifically developed for use in age-related macular degeneration. ${ }^{60} \mathrm{It}$ binds VEGF-A receptors with enhanced affinity and inhibits vasculogenesis. ${ }^{60}$ It is licensed by the FDA for neovascular macular degeneration, macular edema due to retinal vein occlusion, and diabetic retinopathy. ${ }^{59}$

\section{VEGF receptor antagonists/fusion proteins Aflibercept}

Aflibercept (V-Trap; Eylea ${ }^{\mathrm{TM}}$; Regeneron Pharmaceuticals, Tarrytown, NY, USA) is a fusion protein with binding domains for native VEGFR-1 and VEGFR-2. It acts as a decoy receptor irreversibly binding to circulating VEGF-A, VEGF-B, and placental growth factors with significantly higher affinity than bevacizumab. ${ }^{61}$ It is licensed for the treatment of wet age-related macular degeneration and is delivered by intravitreal injection. ${ }^{62}$ In comparison with bevacizumab, ranibizumab, and pegaptanib, Aflibercept has a longer duration of action requiring less frequent administration.

Applications under investigation in oncology include treatment of: metastatic colorectal cancer, ${ }^{63}$ lung adenocarcinoma, ${ }^{53}$ inoperable melanoma, ${ }^{64}$ metastatic urothelial tumors,${ }^{65}$ ovarian cancer, ${ }^{66}$ and recurrent malignant glioma. ${ }^{67}$

Use of Aflibercept in transgenic mice with psoriasis-like lesions led to significant clinical and histological improvement with normalization of the rete ridges and cytoarchitecture, reduced parakeratosis, and vascular hyperplasia. Infiltration of the epidermis by CD8+ lymphocytes was reversed and staining for markers of aberrant epidermal differentiation and vascular inflammation (keratin- 6 and E-selectin) was significantly reduced. ${ }^{25}$

\section{Pegaptanib}

Pegaptanib sodium (Macugen ${ }^{\mathrm{TM}}$; OSI Pharmaceuticals, Melville, NY, USA) is a PEGylated single strand nucleic acid that binds the VEGF165 receptor. It is licensed by the FDA for the treatment of neovascular macular degeneration. It has also been shown to be of benefit in diabetic retinopathy and retinal vein occlusion. ${ }^{68}$

\section{Tyrosine kinase inhibitors (TKIs)}

Kinases are enzymes that exert their biological functions by transferring a phosphate group from high energy donor molecules (such as adenosine triphosphate [ATP]) by phosphorylation resulting in functional changes in target proteins including transcription factors. There are hundreds of human kinases including around 30 tyrosine kinases subdivided into receptor tyrosine kinases and cytoplasmic tyrosine kinases. ${ }^{69}$

TKIs are small molecules which can pass through the plasma membrane and interfere with intracellular tyrosine kinase activity such as those of the VEGFRs. ${ }^{70}$ They not only inhibit VEGFRs, but also act on other kinase receptors, such as those for epidermal growth factor inducing a host of biological effects in addition to inhibition of angiogenesis. ${ }^{71}$

TKIs can be divided into three groups: type I TKIs competitively inhibit binding of ATP in the active conformation of the kinase (eg, sunitinib [Sutent ${ }^{\mathrm{TM}}$; Pfizer, New York, NY, USA]); type II TKIs indirectly compete with ATP by binding to the inactive conformation of the kinase (eg, sorafenib [NexavarTM; Bayer, Leverkusen, Germany]); type III are "covalent inhibitors" that covalently bind cysteines to specific sites on the kinase (eg, vandetanib [Capreslsa $^{\mathrm{TM}}$; AstraZeneca, London, UK]). ${ }^{72}$

TKIs are licensed mainly for the treatment of malignancy, but their use in inflammatory conditions such as rheumatoid $\operatorname{arthritis}^{73}$ and psoriasis ${ }^{74}$ is being investigated.

\section{Sunitinib}

Sunitinib was approved for the treatment of renal cell carcinoma and advanced gastrointestinal stromal tumor in 2007. ${ }^{75}$ It inhibits VEGF kinases and platelet-derived growth factor (PDGF), KIT, and FLT3 receptor tyrosine kinases. ${ }^{76}$ Application to other types of malignant disease including pancreatic neuroendocrine tumor ${ }^{77}$ and uveal melanoma ${ }^{78}$ are underway. 


\section{Sorafenib}

Sorafenib is licensed by the FDA for the treatment of advanced renal cell carcinoma ${ }^{79}$ and inoperable liver carcinoma ${ }^{80}$ Other uses in oncology continue to be explored including application to the treatment of non-small cell lung cancer ${ }^{81}$ and hematological malignancies. ${ }^{82}$ It is an oral multikinase inhibitor which targets not only VEGFRs, but also other kinases including extracellular signal regulated kinase (ERK) and serine/threonine-protein kinase (RAF) kinase. ${ }^{83}$

\section{Efficacy, safety and tolerability}

The most common side effects of systemic administration of bevacizumab are hypertension (36\% of patients) and proteinuria ( $21 \%-64 \%$ of patients). ${ }^{84}$ In cancer patients, an increased risk of hemorrhagic (including gastrointestinal perforation), thrombotic events, and delayed wound healing have also been noted. ${ }^{85}$

In the management of ocular disease, intravitreal bevacizumab is associated with reductions in systemic VEGF-A levels for up to one month after administration. No effect on systemic levels of VEGF-A has been shown after administration of ranibizumab or pegaptanib. ${ }^{86}$ Intravitreal injections of bevacizumab have been associated with acute blood pressure elevation in $0.59 \%$ of patients ${ }^{87}$ but are not associated with increased risk of myocardial infarction, stroke, bleeding, or death. ${ }^{88}$ Intraocular ranibizumab has been associated with an increased risk of stroke and non-ocular bleeding. ${ }^{89}$ Both agents are associated with ocular adverse events including uveitis, retinal tears, retinal detachment, lens damage, and vitreous haemorrhage. ${ }^{90}$

The most commonly observed side effects of Aflibercept therapy in cancer patients include hypertension, fatigue proteinuria, pulmonary hemorrhage, and lymphopenia. ${ }^{63}$

Anti-VEGF mediated hypertension is believed to result from downregulation of nitric oxide synthase, but several other potential pathways (including downregulation of nitric oxide, increased endothelial cell apoptosis, and increased erythropoietin synthesis) may be implicated. ${ }^{91}$

The most common adverse events were similar with sorafenib and sunitinib and included diarrhea, hand-foot syndrome, desquamating skin rash, alopecia, fatigue, and hypertension. ${ }^{92,93}$ These adverse events usually presented early in treatment and are generally of mild to moderate severity. ${ }^{92,94}$

Concern has been raised that TKI, including sorafenib and sunitinib, may be associated with serious adverse cardiovascular effects such as reduced left ventricular function, congestive cardiac failure, and hypertension..$^{95-98}$ Some patients have required intensive intervention including admission to intensive care and/or coronary angiography, pacemaker insertion, and heart surgery. ${ }^{99}$ Recent phase open-label studies in patients with a variety of advanced solid tumors failed to confirm any significant risk of such events; ${ }^{100}$ however, numbers are small and the situation remains under review.

\section{Existing VEGF antagonists and psoriasis}

Improvement in psoriasis in patients being treated for malignancy with VEGF inhibitors has been reported for bevacizumab, ${ }^{21}$ sunitinib, ${ }^{24}$ and sorafenib (Table 1). ${ }^{23}$ These cases are outlined below:

\section{Case I: bevacizumab}

Akman et $\mathrm{al}^{21}$ reported the case of a 60 -year-old man with metastatic colon cancer and a 40-year history of extensive psoriasis affecting $40 \%$ of his body surface area (Psoriasis Area and Severity Index [PASI] 16.8). Following detection of systemic metastases the patient was commenced on systemic therapy with bevacizumab, irinotecan, 5-fluorouracil, and leucovorin. Forty-five days after commencing treatment his psoriasis had significantly improved (PASI 1.4) and his tumor burden had stabilized. Treatment was continued and no relapse of his psoriasis was seen during the 3-month follow-up.

\section{Cases 2 and 3: sunitinib}

Keshtgarpour et $\mathrm{al}^{22}$ reported the first case of sunitinibassociated improvement in co-existing psoriasis during Phase III clinical trials comparing sunitinib to interferon- $\alpha$ $(\mathrm{IFN}-\alpha)$. A male patient with metastatic renal carcinoma and a 20-year history of psoriasis was randomized to receive IFN- $\alpha$. Treatment was continued for 6 months during which time his psoriasis increased in severity forming large confluent plaques across his torso. IFN- $\alpha$ was discontinued and sunitinib commenced 4 weeks later. Introduction of sunitinib was associated with virtual clearance of his psoriasis. During subsequent cycles of sunitinib treatment, exacerbation of psoriasis was noted in the 2 weeks between treatments with progressive improvement during each 4 -week sunitinib cycle.

The second case of sunitinib-associated improvement in psoriasis was reported in 2010 by Narayanan et al. ${ }^{24}$ The second patient was a 60 -year old man with metastatic renal cell carcinoma and a 5-year history of psoriasis. Within 2 weeks of starting sunitinib the patient reported improvement in his psoriasis. As with the first case, cyclical exacerbation was noted during the 2 weeks between treatment cycles. 


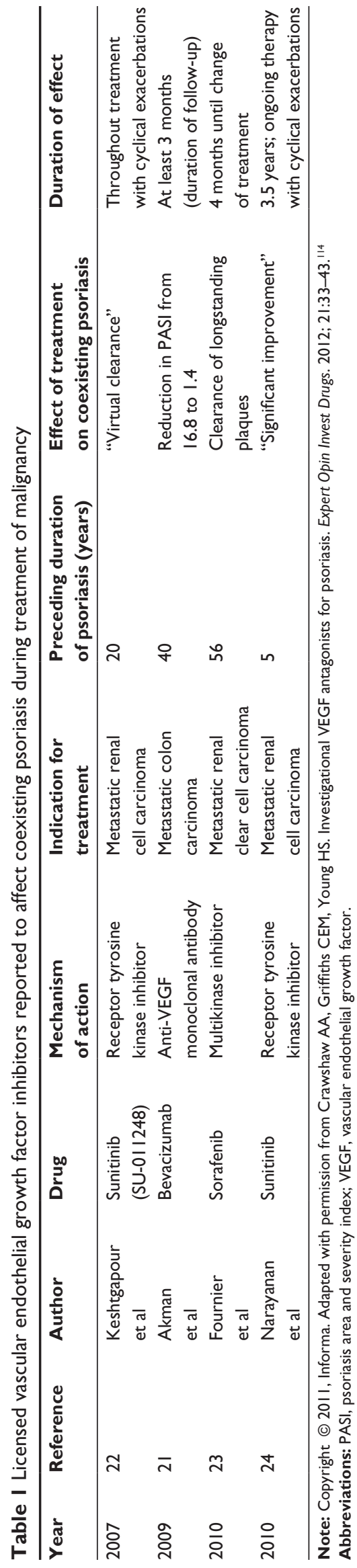

Sustained improvement in his psoriasis continued during the further 3.5 years of sunitinib treatment.

\section{Case 4: sorafenib}

Fournier et al described a 78-year-old man with a 56-year history of psoriasis who was commenced on sorafenib for metastatic renal clear cell carcinoma (hypernephroma). ${ }^{23}$ Prior to treatment, the patient had suffered from a recalcitrant plaque of psoriasis on the posterior mid-thorax measuring $8 \mathrm{~cm}$ by $6 \mathrm{~cm}$. Within 3 weeks of commencing sorafenib, the patient reported a reduction in size of this plaque with complete clearance after 1-month of treatment.

After 4 months of sorafenib, the patient was entirely clear of psoriasis. Unfortunately his hypernephroma became resistant to treatment and sorafenib was discontinued. Within 1-month of ceasing treatment, the psoriatic plaques returned. Interestingly, the patient was subsequently commenced on sunitinib with no reported improvement in his skin disease.

\section{Comments on case reports}

From the cases above it is not possible to directly attribute the clinical improvement in psoriasis with the VEGF inhibitor used for treatment of their malignancy. Stresses, such as systemic illness, are known to be an exacerbating factor in psoriasis. ${ }^{101}$ Removal of such stressors can be associated with improvement of psoriasis ${ }^{102}$ and so it may be that treatment of the underlying malignancy contributed to the improvement in skin disease.

Contrary to this theory is that in three of the four cases the clinical course of psoriasis had been stable prior to commencement of the VEGF inhibitor. In the case reported by Keshtgarpour et al, IFN- $\alpha$ is known to exacerbate psoriasis ${ }^{103}$ and could account for the variability in that patient's skin disease prior to sunitinib therapy. In this case, the significant improvement seen with sorafenib, but not with sunitinib, underlines the heterogeneity of psoriasis as a disease entity. It also suggests that different individuals with psoriasis can display variable responses to tyrosine kinase blockade.

Improvement in psoriasis with VEGF inhibitors is in keeping with our understanding of the role of VEGF in the pathogenesis of psoriasis; however, given the frequency with which VEGF inhibitors are used in oncology one might expect a greater number of cases to have been reported. Isolated reports detailing improvement in psoriasis with other TKIs which do not specifically target VEGFRs (such as lapatinib [Tykerb/Tyverb ${ }^{\mathrm{TM}}$; GlaxoSmithKline, London, UK] ${ }^{104}$ and imatinib [Glivec ${ }^{\mathrm{TM}}$; Novartis, Basel, Switzerland] ${ }^{105}$ are 
also available, but are counter balanced by reports of cutaneous exacerbations of psoriasis with other TKIs. ${ }^{106}$

More research to characterize the nature and frequency of such effects is needed together with a more uniform method for characterizing the degree of baseline skin involvement and degree of improvement.

\section{Emerging therapies in psoriasis}

Investigational VEGF inhibitors for psoriasis mirror those of existing therapies consisting of monoclonal antibodies, fusion proteins, and TKIs (Table 2). None of these agents are currently licensed for treatment of psoriasis, but evidence for potential therapeutic effect is discussed below.

\section{Monoclonal antibodies: G6-3 I, MF- I and DCIOI}

G6-31 is a next generation synthetic monoclonal antibody derived from a phage library that inhibits murine and human VEGF-A with significantly higher affinity than bevacizumab. ${ }^{107}$ In vivo studies using a genetic mouse model of chronic, psoriasis-like skin inflammation with double knockout of the JunB and $c$-Jun alleles ${ }^{108}$ was used to investigate the efficacy of G6-31 in reversing clinical and histological skin changes. Histological manifestations of skin disease included: hyperkeratosis, prominent rete ridges, thickening of the epidermis, parakeratosis, subepidermal vascularization, epidermal microabscess formation, and epidermal infiltration by neutrophils and T-cells similar to those in human psoriasis. ${ }^{109}$

Eight days of systemic administration of G6-31 led to near complete resolution of skin inflammation, scaling, and edema in five out of twelve mice and moderate improvement in a further six mice. Clinical improvement was mirrored by histological normalization, reduced keratinocyte proliferation, and reduced epidermal thickness. ${ }^{109}$

Vascular abnormalities were also reduced with the treated mice showing reduced size of blood and lymphatic vessels and a reduced number of blood vessels. Macrophage and lymphocyte infiltration was inhibited by G6-31 administration and accompanied by reduced levels of cytokines, such as interleukin (IL)-23 and TNF- $\alpha$. Ribonucleic acid (RNA) expression of VEGF-A, VEGFR-1, and VEGFR-2 was also significantly reduced in the treatment group. ${ }^{109}$

MF-1 and DC101 are monoclonal rat antibodies that bind the extracellular domains of mouse VEGFR1 and VEGFR2, respectively. ${ }^{110,111}$ DC101 was originally developed in 1998 as a "proof of concept" tool for investigating the potential of inhibition of angiogenesis in oncology. DC101 does not

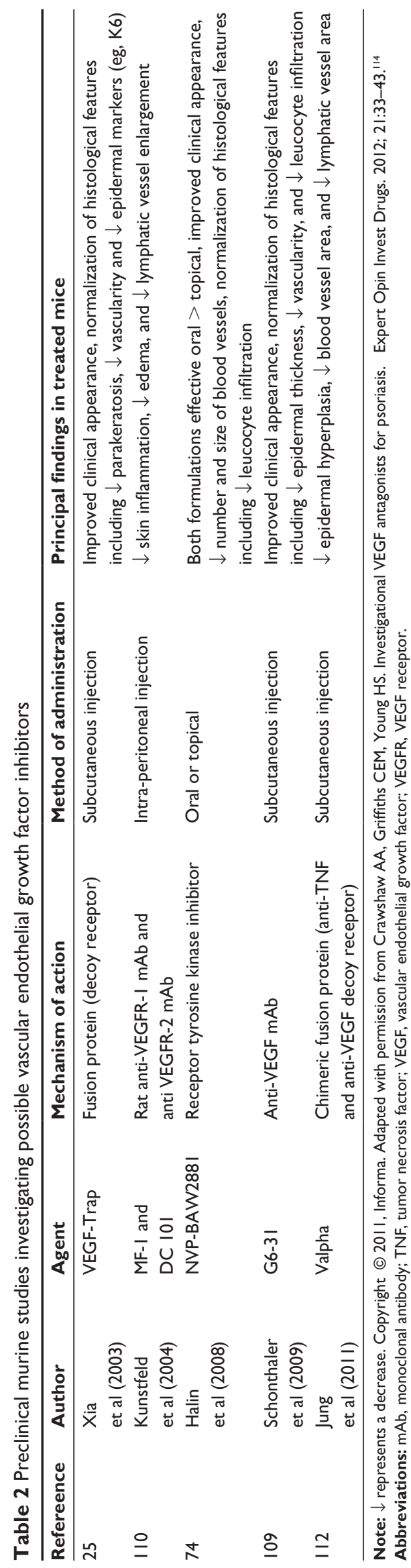


cross react with human VEGFR2 receptors, but can be used to generate synthetic antibodies with in vitro activity against human endothelial cells. ${ }^{111}$

Combined treatment with intra-peritoneal injections of MF-1 and DC101 inhibited experimentally induced skin inflammation in wild-type mice. Treated mice showed reduced skin inflammation, edema, and lymphatic vessel enlargement in comparison to the placebo group. Skin infiltration by $\mathrm{CC} 11 \mathrm{~b}+$ macrophages was also significantly reduced in the dual-treatment and single treatment MF-1 groups. ${ }^{111}$

\section{Fusion proteins: Valpha}

Valpha is a chimeric fusion protein designed to act as a decoy receptor and inhibit both TNF- $\alpha$ and VEGF-A. It contains the VEGF-A-binding domain of VEGFR1, the TNF- $\alpha$-binding domain of TNF receptor 2 (TNFR2) and the Fc domain of human immunoglobulin G1 (IG1).

Valpha has not been tested in humans, however in vitro studies confirmed that Valpha simultaneously binds both TNF- $\alpha$ and VEGF-A with reduced induction of cultured lymphatic endothelial cells and reduced migration of cultured blood endothelial cells. ${ }^{112}$

A 2-week in vivo study using VEGF-A transgenic mice showed a significant reduction in epidermal hyperplasia, blood vessel area, and lymphatic vessel area in comparison to controls treated with either the Fc domain, etanercept (Enbrel $^{\mathrm{TM}}$; Wyeth, Madison, NJ, USA) or Aflibercept.

\section{Tyrosine kinase inhibitors: NVP-BAW288 I}

NVP-BAW2881 targets the tyrosine kinase domain of murine, porcine, and human VEGFR2. It can be administered both orally and topically, but has not been tested in humans. ${ }^{74}$

In vitro studies demonstrated that NVP-BAW2881 inhibited proliferation, migration, and tube formation of human umbilical vein endothelial cells and lymphatic endothelial cells. $^{74}$

In vivo studies in VEGF-A transgenic mice showed that oral and topical administration of NVP-BAW2881 strongly reduced psoriasis-like inflammation in ear skin. Histologically, skin lesions in treated mice showed reduced infiltration by leukocytes, reduced epidermal hyperproliferation, normalization of epidermal keratinocyte differentiation, and fewer vascular abnormalities. Vessels in treated mice were smaller in size and fewer in number. In comparison to control mice, treated mice showed significant improvement in ear swelling, skin inflammation, lymph node enlargement, and skin erythema. Though both modes of administration were effective, systemic administration of NVP-BAW2881 was more potent than topical administration. ${ }^{74}$

Topical NVP-BAW2881 also effectively reduced VEGF-A-induced vascular permeability in the skin of mice and domestic pigs. ${ }^{74}$

\section{Discussion}

Given the widespread use of VEGF inhibitors in oncology and ophthalmology, the very small number of reported incidences of improvement in psoriasis may indicate that existing agents are not targeting the appropriate elements of the angiogenesis pathway in psoriasis. It is also possible that more subtle improvements in mild/moderate psoriasis have gone unnoticed by non-dermatologists.

It is of interest that in animal models dual inhibition of VEGFR-1 and VEGFR-2 (either with combined G6-31 and DC101 or with Aflibercept) achieved clinically relevant results in affected mice. However, caution is needed in interpreting the results from murine models since neither transgenic VEGF-A mice nor double knockout of the JunB and C-Jun mice wholly replicate human psoriasis. It is unsurprising that models that specifically feature upregulation of VEGF as a cause of psoriasis-like inflammatory skin disease may react more impressively to antagonism of this pathway than human skin disease.

New antibodies derived from phage libraries are likely to have less cross-species cross-reactivity than previous antibodies (such as bevacizumab) which are derived from immunizing mice with human VEGF-A. This may aid the translation of research from preclinical investigation into clinical practice. Engineered antibodies, such as the G6 family, display higher affinity for VEGF-A and can bind more securely to VEGFRs overcoming the effects of any in vivo mutations of the VEGF gene and potentially improving efficacy.

It is to be noted that VEGF inhibitors in clinical use are associated with a number of potentially serious side effects including hypertension, left ventricular dysfunction, and gastrointestinal perforation. Such risks require careful consideration in psoriasis populations particularly in light of growing concerns linking psoriasis to increased cardiovascular risk.

In ocular diseases, genetic markers predictive of response to anti-VEGF therapy have been identified ${ }^{113}$ and it is to be hoped that such pharmacogenetic markers will be found in psoriasis in order to identify patients who could benefit 
from VEGF-targeted therapies and maximize effectiveness and safety.

\section{Conclusion}

Though abnormal angiogenesis mediated through VEGFs is a key element in the development of psoriasis, it is a very heterogeneous condition in which many other cytokines and chemokines also play vital roles in disease onset and maintenance.

Given the variety and complexity of the underlying genetic and environmental factors contributing to the pathogenesis of psoriasis, it is unlikely that VEGF antagonism will become an effective approach for therapy in all patients. However, existing data suggests that there is potential for the development of effective agents to act via inhibition of VEGF-A.

A number of exciting avenues for potential treatments are being explored. Of these, TKIs that could be administered topically could have a significant safety (and potentially cost) advantage compared to systemic administration. Further, the use of engineered decoy receptors (like Valpha) to target both VEGF-A and TNF- $\alpha$ provide the potential for dual therapy using only one molecule. Concerns regarding potentially severe side effects associated with use of VEGF inhibitors needs to be carefully addressed in the psoriasis population.

\section{Disclosure}

HSY has acted as a consultant or speaker for Abbott, BiogenIdec, Galderma, Janssen-Cilag, Leo-Pharma, Novartis, Schering-Plough, Stiefel, Teva Pharmaceuticals, and Wyeth/ Pfizer. The authors report no other conflicts of interest in this work.

\section{References}

1. Christophers E. Psoriasis - epidemiology and clinical spectrum. Clin Exp Dermatol. 2001;26(4):314-320.

2. Rapp SR, Feldman SR, Exum ML, Fleischer AB, Reboussin DM. Psoriasis causes as much disability as other major medical diseases. J Am Acad Dermatol. 1999;41(3 Pt 1):401-407.

3. Armstrong AW, Harskamp CT, Armstrong EJ. Psoriasis and metabolic syndrome: a systematic review and meta-analysis of observational studies. J Am Acad Dermatol. 2013;68(4):654-662.

4. Griffiths CE, Barker JN. Pathogenesis and clinical features of psoriasis. Lancet. 2007;370(9583):263-271.

5. Barker JN. The pathophysiology of psoriasis. Lancet. 1991;338(8761): 227-230.

6. Lowes MA, Bowcock AM, Krueger JG. Pathogenesis and therapy of psoriasis. Nature. 2007;445(7130):866-873.

7. Coimbra S, Figueiredo A, Castro E, Rocha-Pereira P, Santos-Silva A. The roles of cells and cytokines in the pathogenesis of psoriasis. Int $J$ Dermatol. 2012;51(4):389-395; quiz 395.

8. Detmar M, Brown LF, Claffey KP, et al. Overexpression of vascular permeability factor/vascular endothelial growth factor and its receptors in psoriasis. J Exp Med. 1994;180(3):1141-1146.
9. Young HS, Summers AM, Bhushan M, Brenchley PE, Griffiths CE. Single-nucleotide polymorphisms of vascular endothelial growth factor in psoriasis of early onset. J Invest Dermatol. 2004;122(1):209-215.

10. Hernández GL, Volpert OV, Iñiguez MA, et al. Selective inhibition of vascular endothelial growth factor-mediated angiogenesis by cyclosporin A: roles of the nuclear factor of activated $\mathrm{T}$ cells and cyclooxygenase 2. J Exp Med. 2001;193(5):607-620.

11. García-Caballero M, Marí-Beffa M, Medina MÁ, Quesada AR. Dimethylfumarate inhibits angiogenesis in vitro and in vivo: a possible role for its antipsoriatic effect? J Invest Dermatol. 2011; 131(6):1347-1355.

12. Meissner M, Doll M, Hrgovic I, et al. Suppression of VEGFR2 expression in human endothelial cells by dimethylfumarate treatment: evidence for anti-angiogenic action. J Invest Dermatol. 2011;131(6):1356-1364.

13. Markham T, Mullan R, Golden-Mason L, et al. Resolution of endothelial activation and down-regulation of Tie2 receptor in psoriatic skin after infliximab therapy. J Am Acad Dermatol. 2006;54(6):1003-1012.

14. Goedkoop AY, Kraan MC, Picavet DI, et al. Deactivation of endothelium and reduction in angiogenesis in psoriatic skin and synovium by low dose infliximab therapy in combination with stable methotrexate therapy: a prospective single-centre study. Arthritis Res Ther. 2004;6(4):R326-R334.

15. Deng H, Yan CL, Hu Y, Xu Y, Liao KH. Photochemotherapy inhibits angiogenesis and induces apoptosis of endothelial cells in vitro. Photodermatol Photoimmunol Photomed. 2004;20(4):191-199.

16. Akman A, Dicle O, Yilmaz F, Coskun M, Yilmaz E. Discrepant levels of vascular endothelial growth factor in psoriasis patients treated with PUVA, Re-PUVA and narrow-band UVB. Photodermatol Photoimmunol Photomed. 2008;24(3):123-127.

17. Kubota Y. Tumor angiogenesis and anti-angiogenic therapy. Keio J Med. 2012;61(2):47-56.

18. Kerr DJ. Targeting angiogenesis in cancer: clinical development of bevacizumab. Nat Clin Pract Oncol. 2004;1(1):39-43.

19. Chang JH, Garg NK, Lunde E, Han KY, Jain S, Azar DT. Corneal neovascularization: an anti-VEGF therapy review. Surv Ophthalmol. 2012;57(5):415-429.

20. Chiang A, Regillo CD. Preferred therapies for neovascular age-related macular degeneration. Curr Opin Ophthalmol. 2011;22(3):199-204.

21. Akman A, Yilmaz E, Mutlu H, Ozdogan M. Complete remission of psoriasis following bevacizumab therapy for colon cancer. Clin Exp Dermatol. 2009;34(5):e202-e204.

22. Keshtgarpour M, Dudek AZ. SU-011248, a vascular endothelial growth factor receptor-tyrosine kinase inhibitor, controls chronic psoriasis. Transl Res. 2007;149(3):103-106.

23. Fournier C, Tisman G. Sorafenib-associated remission of psoriasis in hypernephroma: case report. Dermatol Online J. 2010;16(2):17.

24. Narayanan S, Callis-Duffin K, Batten J, Agarwal N. Improvement of psoriasis during sunitinib therapy for renal cell carcinoma. Am J Med Sci. 2010;339(6):580-581.

25. Xia YP, Li B, Hylton D, Detmar M, Yancopoulos GD, Rudge JS. Transgenic delivery of VEGF to mouse skin leads to an inflammatory condition resembling human psoriasis. Blood. 2003;102(1):161-168.

26. Folkman J, Shing Y. Angiogenesis. J Biol Chem. 1992;267(16): 10931-10934.

27. Folkman J. The role of angiogenesis in tumor growth. Semin Cancer Biol. 1992;3(2):65-71.

28. Bianchi E, Scarinci F, Grande C, et al. Immunohistochemical profile of VEGF, TGF- $\beta$ and PGE2 in human pterygium and normal conjunctiva: experimental study and review of the literature. Int J Immunopathol Pharmacol. 2012;25(3):607-615.

29. Brenchley PE. Angiogenesis in inflammatory joint disease: a target for therapeutic intervention. Clin Exp Immunol. 2000;121(3):426-429.

30. Ferrara N. Vascular endothelial growth factor: basic science and clinical progress. Endocr Rev. 2004;25(4):581-611.

31. Kiselyov A, Balakin KV, Tkachenko SE. VEGF/VEGFR signalling as a target for inhibiting angiogenesis. Expert Opin Investig Drugs. 2007;16(1):83-107. 
32. Shinkaruk S, Bayle M, Laïn G, Déléris G. Vascular endothelial cell growth factor (VEGF), an emerging target for cancer chemotherapy. Curr Med Chem Anticancer Agents. 2003;3(2):95-117.

33. Clauss M, Gerlach M, Gerlach H, et al. Vascular permeability factor: a tumor-derived polypeptide that induces endothelial cell and monocyte procoagulant activity, and promotes monocyte migration. J Exp Med. 1990;172(6):1535-1545.

34. Ferrara N, Gerber HP, LeCouter J. The biology of VEGF and its receptors. Nat Med. 2003;9(6):669-676.

35. Houck KA, Leung DW, Rowland AM, Winer J, Ferrara N. Dual regulation of vascular endothelial growth factor bioavailability by genetic and proteolytic mechanisms. J Biol Chem. 1992;267(36):26031-26037.

36. Takahashi S. Vascular endothelial growth factor (VEGF), VEGF receptors and their inhibitors for antiangiogenic tumor therapy. Biol Pharm Bull. 2011;34(12):1785-1788.

37. Carmeliet P, Ferreira V, Breier G, et al. Abnormal blood vessel development and lethality in embryos lacking a single VEGF allele. Nature. 1996;380(6573):435-439.

38. Brogan IJ, Khan N, Isaac K, Hutchinson JA, Pravica V, Hutchinson IV. Novel polymorphisms in the promoter and 5' UTR regions of the human vascular endothelial growth factor gene. Hum Immunol. 1999;60(12):1245-1249.

39. Watson CJ, Webb NJ, Bottomley MJ, Brenchley PE. Identification of polymorphisms within the vascular endothelial growth factor (VEGF) gene: correlation with variation in VEGF protein production. Cytokine. 2000;12(8):1232-1235.

40. Young HS, Summers AM, Read IR, et al. Interaction between genetic control of vascular endothelial growth factor production and retinoid responsiveness in psoriasis. J Invest Dermatol. 2006; 126(2):453-459.

41. Bhushan M, McLaughlin B, Weiss JB, Griffiths CE. Levels of endothelial cell stimulating angiogenesis factor and vascular endothelial growth factor are elevated in psoriasis. Br J Dermatol. 1999;141(6): 1054-1060.

42. Andrys C, Borska L, Pohl D, Fiala Z, Hamakova K, Krejsek J. Angiogenic activity in patients with psoriasis is significantly decreased by Goeckerman's therapy. Arch Dermatol Res. 2007;298(10):479-483.

43. Creamer D, Allen M, Jaggar R, Stevens R, Bicknell R, Barker J. Mediation of systemic vascular hyperpermeability in severe psoriasis by circulating vascular endothelial growth factor. Arch Dermatol. 2002;138(6):791-796.

44. Matsumura Y, Ananthaswamy HN. Short-term and long-term cellular and molecular events following UV irradiation of skin: implications for molecular medicine. Expert Rev Mol Med. 2002;4(26):1-22.

45. Yano K, Kadoya K, Kajiya K, Hong YK, Detmar M. Ultraviolet B irradiation of human skin induces an angiogenic switch that is mediated by upregulation of vascular endothelial growth factor and by downregulation of thrombospondin-1. Br J Dermatol. 2005;152(1):115-121.

46. Bielenberg DR, Bucana CD, Sanchez R, Donawho CK, Kripke ML, Fidler IJ. Molecular regulation of UVB-induced cutaneous angiogenesis. J Invest Dermatol. 1998;111(5):864-872.

47. Arbiser JL. Fumarate esters as angiogenesis inhibitors: key to action in psoriasis? J Invest Dermatol. 2011;131(6):1189-1191.

48. Giraudo E, Primo L, Audero E, et al. Tumor necrosis factor-alpha regulates expression of vascular endothelial growth factor receptor-2 and of its co-receptor neuropilin-1 in human vascular endothelial cells. J Biol Chem. 1998;273(34):22128-22135.

49. Brotas AM, Cunha JM, Lago EH, Machado CC, Carneiro SC. Tumor necrosis factor-alpha and the cytokine network in psoriasis. An Bras Dermatol. 2012;87(5):673-681; quiz 682.

50. Tobin AM, Kirby B. TNF alpha inhibitors in the treatment of psoriasis and psoriatic arthritis. BioDrugs. 2005;19(1):47-57.

51. Korpanty G, Smyth E. Anti-VEGF strategies - from antibodies to tyrosine kinase inhibitors: background and clinical development in human cancer. Curr Pharm Des. 2012;18(19):2680-2701.

52. Veritti D, Sarao V, Lanzetta P. Neovascular age-related macular degeneration. Ophthalmologica. 2012;227 Suppl 1:11-20.
53. Aggarwal C, Somaiah N, Simon G. Antiangiogenic agents in the management of non-small cell lung cancer: where do we stand now and where are we headed? Cancer Biol Ther. 2012;13(5): 247-263.

54. Presta LG, Chen H, O’Connor SJ, et al. Humanization of an anti-vascular endothelial growth factor monoclonal antibody for the therapy of solid tumors and other disorders. Cancer Res. 1997;57(20):4593-4599.

55. Cohen MH, Gootenberg J, Keegan P, Pazdur R. FDA drug approval summary: bevacizumab plus FOLFOX4 as second-line treatment of colorectal cancer. Oncologist. 2007;12(3):356-361.

56. Cohen MH, Gootenberg J, Keegan P, Pazdur R. FDA drug approval summary: bevacizumab (Avastin) plus Carboplatin and Paclitaxel as first-line treatment of advanced/metastatic recurrent nonsquamous non-small cell lung cancer. Oncologist. 2007;12(6): 713-718.

57. Summers J, Cohen MH, Keegan P, Pazdur R. FDA drug approval summary: bevacizumab plus interferon for advanced renal cell carcinoma. Oncologist. 2010;15(1):104-111.

58. Aghajanian C, Blank SV, Goff BA, et al. OCEANS: a randomized, double-blind, placebo-controlled phase III trial of chemotherapy with or without bevacizumab in patients with platinum-sensitive recurrent epithelial ovarian, primary peritoneal, or fallopian tube cancer. J Clin Oncol. 2012;30(17):2039-2045.

59. Saeed MU, Gkaragkani E, Ali K. Emerging roles for antiangiogenesis factors in management of ocular disease. Clin Ophthalmol. 2013;6:533-543.

60. Ferrara N, Damico L, Shams N, Lowman H, Kim R. Development of ranibizumab, an anti-vascular endothelial growth factor antigen binding fragment, as therapy for neovascular age-related macular degeneration. Retina (Philadelphia, Pa). 2006;26(8):859-870.

61. Holash J, Davis S, Papadopoulos N, et al. VEGF-Trap: a VEGF blocker with potent antitumor effects. Proc Natl Acad Sci U S A. 2002;99(17):11393-11398.

62. Stewart MW, Grippon S, Kirkpatrick P. Aflibercept. Nat Rev Drug Discov. 2012;11(4):269-270.

63. Wang TF, Lockhart AC. Aflibercept in the treatment of metastatic colorectal cancer. Clin Med Insights Oncol. 2012;6:19-30.

64. Tarhini AA, Frankel P, Margolin KA, et al. Aflibercept (VEGF Trap) in inoperable stage III or stage iv melanoma of cutaneous or uveal origin. Clin Cancer Res. 2011;17(20):6574-6581.

65. Twardowski P, Stadler WM, Frankel P, et al. Phase II study of Aflibercept (VEGF-Trap) in patients with recurrent or metastatic urothelial cancer, a California Cancer Consortium Trial. Urology. 2010;76(4):923-926.

66. Amini A, Masoumi Moghaddam S, Morris DL, Pourgholami MH. Utility of vascular endothelial growth factor inhibitors in the treatment of ovarian cancer: from concept to application. J Oncol. 2012;2012:540791.

67. de Groot JF, Lamborn KR, Chang SM, et al. Phase II study of aflibercept in recurrent malignant glioma: a North American Brain Tumor Consortium study. J Clin Oncol. 2011;29(19):2689-2695.

68. Apte RS. Pegaptanib sodium for the treatment of age-related macular degeneration. Expert Opin Pharmacother. 2008;9(3):499-508.

69. Manning G, Whyte DB, Martinez R, Hunter T, Sudarsanam S. The protein kinase complement of the human genome. Science. 2002;298(5600):1912-1934.

70. Imai K, Takaoka A. Comparing antibody and small-molecule therapies for cancer. Nat Rev Cancer. 2006;6(9):714-727.

71. Morabito A, Piccirillo MC, Falasconi F, et al. Vandetanib (ZD6474), a dual inhibitor of vascular endothelial growth factor receptor (VEGFR) and epidermal growth factor receptor (EGFR) tyrosine kinases: current status and future directions. Oncologist. 2009;14(4):378-390.

72. Ribatti D. Tyrosine kinase inhibitors as antiangiogenic drugs in multiple myeloma. Pharmaceuticals. 2010;3:1225-1231.

73. O'Shea JJ, Laurence A, McInnes IB. Back to the future: oral targeted therapy for RA and other autoimmune diseases. Nat Rev Rheumatol. 2013;9(3):173-182. 
74. Halin C, Fahrngruber H, Meingassner JG, et al. Inhibition of chronic and acute skin inflammation by treatment with a vascular endothelial growth factor receptor tyrosine kinase inhibitor. Am J Pathol. 2008;173(1):265-277.

75. Rock EP, Goodman V, Jiang JX, et al. Food and Drug Administration drug approval summary: Sunitinib malate for the treatment of gastrointestinal stromal tumor and advanced renal cell carcinoma. Oncologist. 2007;12(1):107-113.

76. Mendel DB, Laird AD, Xin X, et al. In vivo antitumor activity of SU11248, a novel tyrosine kinase inhibitor targeting vascular endothelial growth factor and platelet-derived growth factor receptors: determination of a pharmacokinetic/pharmacodynamic relationship. Clin Cancer Res. 2003;9(1):327-337.

77. Raymond E, Hammel P, Dreyer C, et al. Sunitinib in pancreatic neuroendocrine tumors. Target Oncol. 2012;7(2):117-125.

78. Velho TR, Kapiteijn E, Jager MJ. New therapeutic agents in uveal melanoma. Anticancer Res. 2012;32(7):2591-2598.

79. Escudier B, Eisen T, Stadler WM, et al; TARGET Study Group. Sorafenib in advanced clear-cell renal-cell carcinoma. $N$ Engl J Med. 2007;356(2):125-134.

80. Lang L. FDA approves sorafenib for patients with inoperable liver cancer. Gastroenterology. 2008;134(2):379.

81. Zhang J, Gold KA, Kim E. Sorafenib in non-small cell lung cancer. Expert Opin Investig Drugs. 2012;21(9):1417-1426.

82. Zauli G, Voltan R, Tisato V, Secchiero P. State of the art of the therapeutic perspective of sorafenib against hematological malignancies. Curr Med Chem. 2012;19(28):4875-4884.

83. Wilhelm SM, Adnane L, Newell P, Villanueva A, Llovet JM, Lynch M. Preclinical overview of sorafenib, a multikinase inhibitor that targets both Raf and VEGF and PDGF receptor tyrosine kinase signaling. Mol Cancer Ther. 2008;7(10):3129-3140.

84. Zhu X, Wu S, Dahut WL, Parikh CR. Risks of proteinuria and hypertension with bevacizumab, an antibody against vascular endothelial growth factor: systematic review and meta-analysis. Am J Kidney Dis 2007;49(2):186-193.

85. van Heeckeren WJ, Sanborn SL, Narayan A, et al. Complications from vascular disrupting agents and angiogenesis inhibitors: aberrant control of hemostasis and thrombosis. Curr Opin Hematol. 2007; 14(5):468-480.

86. Zehetner C, Kirchmair R, Huber S, Kralinger MT, Kieselbach GF Plasma levels of vascular endothelial growth factor before and after intravitreal injection of bevacizumab, ranibizumab and pegaptanib in patients with age-related macular degeneration, and in patients with diabetic macular oedema. Br J Ophthalmol. 2013;97(4):454-459.

87. Wu L, Martínez-Castellanos MA, Quiroz-Mercado H, et al; Pan American Collaborative Retina Group (PACORES). Twelve-month safety of intravitreal injections of bevacizumab (Avastin): results of the Pan-American Collaborative Retina Study Group (PACORES). Graefes Arch Clin Exp Ophthalmol. 2008;246(1):81-87.

88. Curtis LH, Hammill BG, Schulman KA, Cousins SW. Risks of mortality, myocardial infarction, bleeding, and stroke associated with therapies for age-related macular degeneration. Arch Ophthalmol. 2010;128(10):1273-1279.

89. Gillies MC, Wong TY. Ranibizumab for neovascular age-related macular degeneration. $N$ Engl J Med. 2007;356(7):748-9; author reply 749.

90. Schmucker C, Ehlken C, Agostini HT, et al. A safety review and meta-analyses of bevacizumab and ranibizumab: off-label versus goldstandard. PLoS One. 2012;7(8):e42701.

91. Stewart MW. The expanding role of vascular endothelial growth factor inhibitors in ophthalmology. Mayo Clin Proc. 2012;87(1):77-88.

92. Hutson TE, Bellmunt J, Porta C, et al; Sorafenib TARGET Clinical Trial Group. Long-term safety of sorafenib in advanced renal cell carcinoma: follow-up of patients from phase III TARGET. Eur $J$ Cancer. 2010;46(13):2432-2440.

93. Adams VR, Leggas M. Sunitinib malate for the treatment of metastatic renal cell carcinoma and gastrointestinal stromal tumors. Clin Ther. 2007;29(7):1338-1353.
94. Procopio G, Bellmunt J, Dutcher J, et al. Sorafenib tolerability in elderly patients with advanced renal cell carcinoma: results from a large pooled analysis. Br J Cancer. 2013;108(2):311-318.

95. Khakoo AY, Kassiotis CM, Tannir N, et al. Heart failure associated with sunitinib malate: a multitargeted receptor tyrosine kinase inhibitor. Cancer. 2008;112(11):2500-2508.

96. Machiels JP, Bletard N, Pirenne P, Jacquet L, Bonbled F, Duck L. Acute cardiac failure after sunitinib. Ann Oncol. 2008;19(3):597-599.

97. Telli ML, Witteles RM, Fisher GA, Srinivas S. Cardiotoxicity associated with the cancer therapeutic agent sunitinib malate. Ann Oncol. 2008;19(9):1613-1618.

98. Di Lorenzo G, Autorino R, Bruni G, et al. Cardiovascular toxicity following sunitinib therapy in metastatic renal cell carcinoma: a multicenter analysis. Ann Oncol. 2009;20(9):1535-1542.

99. Schmidinger M, Zielinski CC, Vogl UM, et al. Cardiac toxicity of sunitinib and sorafenib in patients with metastatic renal cell carcinoma. $J$ Clin Oncol. 2008;26(32):5204-5212.

100. Tolcher AW, Appleman LJ, Shapiro GI, et al. A phase I open-label study evaluating the cardiovascular safety of sorafenib in patients with advanced cancer. Cancer Chemother Pharmacol. 2011;67(4):751-764.

101. Al'Abadie MS, Kent GG, Gawkrodger DJ. The relationship between stress and the onset and exacerbation of psoriasis and other skin conditions. Br J Dermatol. 1994;130(2):199-203.

102. Fortune DG, Richards HL, Griffiths CE. Psychologic factors in psoriasis: consequences, mechanisms, and interventions. Dermatol Clin. 2005;23(4):681-694.

103. Collamer AN, Battafarano DF. Psoriatic skin lesions induced by tumor necrosis factor antagonist therapy: clinical features and possible immunopathogenesis. Semin Arthritis Rheum. 2010;40(3): 233-240.

104. Wierzbicka E, Tourani JM, Guillet G. Improvement of psoriasis and cutaneous side-effects during tyrosine kinase inhibitor therapy for renal metastatic adenocarcinoma. A role for epidermal growth factor receptor (EGFR) inhibitors in psoriasis? $\mathrm{Br} J$ Dermatol. 2006;155(1):213-214.

105. Miyagawa S, Fujimoto H, Ko S, Hirota S, Kitamura Y. Improvement of psoriasis during imatinib therapy in a patient with a metastatic gastrointestinal stromal tumour. Br J Dermatol. 2002;147(2):406-407.

106. Zorzou MP, Stratigos A, Efstathiou E, Bamias A. Exacerbation of psoriasis after treatment with an EGFR tyrosine kinase inhibitor. Acta Derm Venereol. 2004;84(4):308-309.

107. O'Connor JP, Carano RA, Clamp AR, et al. Quantifying antivascular effects of monoclonal antibodies to vascular endothelial growth factor: insights from imaging. Clin Cancer Res. 2009;15(21):6674-6682.

108. Zenz R, Eferl R, Kenner L, et al. Psoriasis-like skin disease and arthritis caused by inducible epidermal deletion of Jun proteins. Nature. 2005;437(7057):369-375.

109. Schonthaler HB, Huggenberger R, Wculek SK, Detmar M, Wagner EF. Systemic anti-VEGF treatment strongly reduces skin inflammation in a mouse model of psoriasis. Proc Natl Acad Sci USA. 2009;106(50):21264-21269.

110. Kunstfeld R, Hirakawa S, Hong YK, et al. Induction of cutaneous delayed-type hypersensitivity reactions in VEGF-A transgenic mice results in chronic skin inflammation associated with persistent lymphatic hyperplasia. Blood. 2004;104(4):1048-1057.

111. Witte L, Hicklin DJ, Zhu Z, et al. Monoclonal antibodies targeting the VEGF receptor-2 (Flk1/KDR) as an anti-angiogenic therapeutic strategy. Cancer Metastasis Rev. 1998;17(2):155-161.

112. Jung K, Lee D, Lim HS, et al. Double anti-angiogenic and anti-inflammatory protein Valpha targeting VEGF-A and TNF-alpha in retinopathy and psoriasis. J Biol Chem. 2011;286(16):14410-14418.

113. Agosta E, Lazzeri S, Orlandi P, et al. Pharmacogenetics of antiangiogenic and antineovascular therapies of age-related macular degeneration. Pharmacogenomics. 2012;13(9):1037-1053.

114. Crawshaw AA, Griffiths CEM, Young HS. Investigational VEGF antagonists for psoriasis. Expert Opin Invest Drugs. 2012;21: $33-43$. 


\section{Publish your work in this journal}

Clinical, Cosmetic and Investigational Dermatology is an international, peer-reviewed, open access, online journal that focuses on the latest clinical and experimental research in all aspects of skin disease and cosmetic interventions. All areas of dermatology will be covered; contributions will be welcomed from all clinicians and

basic science researchers globally. This journal is indexed on CAS

The manuscript management system is completely online and includes

a very quick and fair peer-review system, which is all easy to use. Visit http://www.dovepress.com/testimonials.php to read real quotes from published authors.

Submit your manuscript here: http://www.dovepress.com/clinical-cosmetic-and-investigational-dermatology-journal 